\title{
Schluss und Ausblick
}

Das Burnout-Syndrom kann schnell aus dem öffentlichen Blick verschwinden und in Vergessenheit geraten. Wie die Erfahrung zeigt, dominieren immer neuere und aktuellere Themen, wie etwa der Umweltaktivismus von Greta Thunberg, der Brexit, die US-Wahlen oder die Covid-19-Pandemie, die mediale Präsenz und bestimmen den Fokus gesellschaftlicher, politischer, finanzieller, aber auch wissenschaftlicher Interessen. Nichtdestotrotz tritt dieses Thema zwar weniger medial, dafür aber deutlich dringender auf, was auch weitere Studien belegen (vgl. Brendel \& Martus 2018). Das Vorhaben, dem Burnout-Syndrom, und vor allem der BurnoutPrävention, einen Spiegel vorzuhalten zeigte, wie die Beziehungen der Subjekte zu sich selbst und zu den anderen geschwächt bis unterdrückt werden. Diese Beziehungen wiederherzustellen, schonend auszutarieren und in Einklang mit Umwelt, Kultur, Technik und Gesellschaft zu bringen, kann und muss von Prozessen kritischer Selbst- und Gesellschaftsprüfung begleitet werden, damit der Raum für das Denken politischer Handlungsfähigkeit stets offenbleibt.

Betrachtet aus der politisch-theoretischen Perspektive, beleuchtete das Projekt die asymmetrische Verteilung der Machtverhältnisse, die durch Hervorbringung leistungsstarker, aber gleichzeitig auch gefährdungsbewusster und präventiver, Subjektformen den Gesellschaftskörper spalten. Die Spaltungen betreffen nicht die Bevölkerung als eine Summe von Individuen, sondern, wie die Analyse nahelegte, finden im Inneren-in dem Bezug des Subjekts zu sich selbst—statt. Dieser Konstitutionsakt einer Subjektform kollidiert dann mit dem genuin ")politischen Institutionsakt « (Marchart 2013: 430 [Herv. i. O.]) einer demokratischen Gesellschaft und erweckt den Anschein einer alternativlosen, weil zwangsläufigen Entwicklung. Entgegen der Vorstellung, die Gesellschaft entwickele sich nach ihren besten Möglichkeiten und das Burnout-Syndrom sei dafür der mildeste Preis, zeigte diese Forschung, wie trügerisch und kontingent jegliche Regierungstechnologien, inclusive Prävention, sind. Was demnach mehr in den Vordergrund rücken muss, ist das Wissen über die Gegenwärtigkeit und Wandelbarkeit der Machtund Regierungsverhältnisse, ebenso wie die Erfahrung einer kollektivverschuldeten Unmündigkeit bzw. eines Burnout-Syndroms neoliberaler Gesellschaft. 
Zuallerletzt sollen am Ende der durchgeführten Analysen, Kritiken und Überlegungen zum neoliberalen Dispositiv der Prävention einige Bausteine für die weitere Forschung geliefert werden. Diese lassen sich in drei Teilen betrachten:

- Erstens orientiert sich die Kritik des Regierens und Regiert-Werdens in den poststrukturalistischen Ansätzen vor allem an dem Ergründen der Rationalität, durch die bestimmte Elemente wie Diskurse oder nicht-diskursive Praktiken zu einem funktionierenden Regime werden. Eine solche Rationalität wird dann auf ihre Logiken und inhärente Herrschaftsmechanismen hinterfragt. Dasselbe betrifft auch den Präventionsdispositiv. Allerdings, was in dieser Hinsicht stärker in den Fokus geraten kann, ist der Blick auf die Emotionalität des Regierens (Han 2015). ${ }^{1}$ Dabei soll nicht nur die Frage gestellt werden, wie Emotionen instrumentalisiert und zum Zweck des Regierens eingesetzt werden, sondern auch die Frage, wie sie als Nebeneffekte des Regierens erscheinen. Mit anderen Worten, welche Gefühls- und Gemütslage erzeugt ein politisches Regime, wie bspw. der Neoliberalismus? Wie werden diese erkenn- und spürbar? Welche emotionalen Reaktionen kommen den Subjekten normal, welche dagegen seltsam vor? Wie sollen sich im neoliberalen Regime die ausgebrannten Subjekte als emotionale Subjekte inszenieren? Mit Scham, Ohnmacht und Müdigkeit oder als Subjekte mit neuen Möglichkeiten der Lebensgestaltung?

- Des Weiteren richtet sich vor allem die neomarxistische und linksorientierte Kritik des Regierens auf die Möglichkeiten und Organisation des Widerstands, der von globalen Bewegungen einer Klassenrevolution bis hin zu lokalen Mikrowiderständen reicht. Worauf sich die Aufmerksamkeit emanzipatorischer Bewegungen verstärkt richten kann, ist die Exposition individueller Erfahrungen als gesellschaftliche Zustände (vgl. Eribon [2013] 2017). Die verschiedensten gesellschaftlichen Wandlungen schlagen sich in den individuellen Erfahrungswelten nieder und können aus diesen wieder extrapoliert und als Mikrokosmen politischer Verhältnisse gedeutet werden. Hiermit können den Subjekten Machtpositionen zugänglich gemacht werden, die für sie bisher unbewusst oder ausgeblendet waren. So kann sich bspw. in einer Liebesbeziehung zwischen zwei Personen unterschiedlicher Herkunft und sozialer Lage ein Aufklärungs-, Emanzipations- und Gleichstellungsprozess vollziehen, der nicht nur das (Zusammen-)Leben dieser Individuen betrifft, sondern der zugleich die unterschiedlichen gesellschaftlichen Normen, unsichtbaren Steuerungsmechanismen und unbewussten oppressiven Haltungen in Frage stellt,

1 Der affective turn in den Geisteswissenschaften wurde bereits signalisiert (vgl. Hochschild [1983] 2003; Illouz 2006; Clough \& Halley 2007; Liljeström \& Paasonen 2010; Mouffe 2014; Penz \& Sauer 2016). 
eventuell bricht und neujustiert. Ein individuelles Erleiden und Erproben dieser Erfahrungen konstituiert neue Ausgangslagen für das Nachdenken über gültige soziale und politische Verhältnisse und rüstet die Individuen mit Macht und Verantwortung für kollektive Veränderungen aus.

- Schließlich richtet sich die Kritik des Regierens auf die Untersuchung und das Hinterfragen von Subjektformierungen. In diesem Zusammenhang dreht sich die Kritik darum, inwiefern die Subjekte eine Autonomie besitzen und wie sich diese begründen lässt (vgl. Cohen 2012). Vor allem im Zustand einer totalen Erschöpfung kommt dieser Frage eine stärkere Bedeutung zu. Eine mögliche Perspektive der Kritik bietet sich in Form von Verhalten an, das das politische Regime provoziert und zu durchbrechen sucht, wodurch die Autonomie der Subjekte erprobt werden kann (vgl. Rancière 2014). Außerdem können sich Subjekte bewusst gegen Konsum, Lust und Überfluss an Optionen und Gütern entscheiden und sich in Askese und Selbstbeschränkung üben, was ihnen ein Gefühl der Wahlfreiheit und Selbstbeherrschung vermitteln soll (Gronemeyer 1998). Subjektformierungen stellen allerdings ein analytisches Instrument, nicht eine ontologische Konstante dar. Ein Subjekt zu werden im analytischen Sinne heißt noch längst nicht, dass das Individuum unfrei geworden ist. Stattdessen werden diese Instrumente als kontrastgebende Substanz eingesetzt, um die Machtverhältnisse sichtbar zu machen. Die Aufgabe besteht also nicht im Abgrenzen der Autonomieräume, sondern im Schärfen der analytischen Kategorien, die diese Räume freisetzen können. 
